\title{
Frailescano: nueva variedad de frijol negro opaco para Chiapas
}

\author{
Bernardo Villar-Sánchez ${ }^{1 \S}$ \\ Oscar Hugo Tosquy-Valle ${ }^{2}$ \\ Ernesto López-Salinas ${ }^{2}$ \\ Francisco Javier Cruz-Chávez ${ }^{1}$ \\ Jorge Alberto Acosta-Gallegos ${ }^{3}$ \\ ${ }^{1}$ Campo Experimental Centro de Chiapas-INIFAP. Carretera Ocozocoautla-Cintalapa km 3.0, \\ Ocozocoautla, Chiapas, México. CP. 29140. ${ }^{2}$ Campo Experimental Cotaxtla-INIFAP. Carretera Federal \\ Veracruz-Córdoba km 34.5, Medellín de Bravo, Veracruz, México. CP. 91700. \\ (tosquy.oscar@inifap.gob.mx; lopez.ernesto@inifap.gob.mx). ${ }^{3}$ Campo Experimental Bajío-INIFAP. \\ Carretera Celaya-San Miguel de Allende km 6.5, Celaya, Guanajuato, México. CP. 38000. \\ (acosta.jorge@inifap.gob.mx). \\ ${ }^{\S}$ Autor para correspondencia: villar.bernardo@inifap.gob.mx.
}

\section{Resumen}

En Chiapas, México, la productividad de frijol es afectada por la incidencia de mosaico amarillo dorado, la acidez de los suelos y el uso de variedades criollas de bajo rendimiento y susceptibles a enfermedades. Para contribuir a solucionar esta problemática en 2013 se generó la variedad Frailescano. Durante 2011 y 2012, la línea SCN 6 que dio origen a la variedad Frailescano se evaluó en ocho ambientes de Chiapas. El rendimiento promedio general de la variedad Frailescano fue de $1150 \mathrm{~kg} \mathrm{ha}^{-1}$, similar al de Negro Papaloapan y significativamente superior al de Negro Comapa. En 2012 se evaluó su reacción al mosaico amarillo dorado en condiciones de campo. La variedad Frailescano mostró $13 \%$ de incidencia, en tanto que, las variedades Negro Papaloapan y Negro Comapa presentaron incidencias de 40 y 23\%, respectivamente. En ese mismo año, se evaluó su respuesta productiva en suelo ácido, sin y con aplicación de $1 \mathrm{t} \mathrm{ha}^{-1}$ de cal agrícola. En ambas condiciones de suelo, la variedad Frailescano superó en más de 96\% el rendimiento del Criollo Veracruzano y mostró menor reducción de rendimiento, debido al estrés por suelo ácido. De 2014 a 2016 la variedad Frailescano se validó en terrenos de agricultores, bajo riego y humedad residual; en ambos casos, superó en rendimiento al criollo Vaina Negra. El rendimiento promedio general de Frailescano fue de $1438 \mathrm{~kg} \mathrm{ha}^{-1}$, significativamente superior (51.1\%) al del criollo regional. En 2013, Frailescano se registró en el Servicio Nacional de Inspección y Certificación de Semillas, para su uso comercial.

Palabras clave: Phaseolus vulgaris L., adaptabilidad, BGYMV, cultivar, rendimiento.

Recibido: noviembre de 2020

Aceptado: diciembre de 2020 
El estado de Chiapas ocupa el quinto lugar en producción de frijol a nivel nacional, durante 2018 se sembraron 112319 ha, de las que se produjeron 64,393 t de grano, principalmente de color negro opaco, con un rendimiento promedio de $575 \mathrm{~kg} \mathrm{ha}^{-1}$ (SIAP, 2020). El bajo rendimiento es debido a que el cultivo es afectado por factores bióticos, abióticos y técnicos entre los que destacan: la incidencia de enfermedades como el mosaico amarillo dorado del frijol (BGYMV), la cual puede provocar pérdidas significativas en el rendimiento de grano, sobre todo cuando se presenta durante la fase vegetativa del cultivo (López et al., 2003; Tosquy et al., 2012; Villar et al., 2017), la siembra de frijol en suelos de baja fertilidad y ácidos, que limitan el desarrollo y productividad del cultivo, por una baja disponibilidad de nutrientes (Zetina et al., 2002; López et al., 2006) y el uso de variedades criollas de bajo rendimiento, limitada adaptación y susceptibles al BGYMV (Villar et al., 2013).

Una alternativa viable para contribuir a solucionar esta problemática es desarrollar variedades mejoradas de alto potencial de rendimiento y con tolerancia a los principales factores limitantes (Rosas et al., 2003). El Programa de Frijol del Instituto Nacional de Investigaciones Forestales, Agrícolas y Pecuarias (INIFAP) en Chiapas, ha desarrollado variedades de frijol negro con las características agronómicas superiores, las cuales algunas de ellas están siendo utilizadas por los productores (Villar et al., 1993; 2003; 2010; 2011).

En 2013 se obtuvo el registro de la variedad Frailescano en el servicio nacional de inspección y certificación de semillas (SNICS), para su uso comercial en las áreas tropicales del estado de Chiapas y regiones con condiciones similares del sureste de México (Villar et al., 2013). El objetivo de este trabajo es dar a conocer el origen y proceso de obtención de esta variedad, sus principales características agronómicas, su comportamiento productivo en diferentes ambientes de evaluación y las condiciones agroecológicas adecuadas para su siembra.

\section{Origen y proceso de obtención}

Esta variedad se originó de la cruza triple (NCB 228/RCB 224) $\mathrm{F}_{1} / / \mathrm{SXB} 244$, la cual se realizó en el Centro Internacional de Agricultura Tropical (CIAT), en Cali, Colombia. El proceso de desarrollo inició en $F_{1}$ como 'gameto' o planta $F_{1}$ cosechada en forma individual, para crear una familia única derivada de $\mathrm{F}_{1}$ y luego continuó con $\mathrm{F}_{2}$ en la que se realizó selección en condiciones de sequía en Palmira, Colombia. Las plantas $\mathrm{F}_{3}$ se establecieron en Popayán, Colombia, donde se inocularon con Colletotrichum lindemuthianum y se seleccionaron plantas individuales resistentes a la antracnosis causada por este hongo.

$\mathrm{La} \mathrm{F}_{4}$ se sembró en Santander de Quilichao, Colombia, en un suelo ácido, con bajo contenido de fósforo e inoculado con el hongo Phaeoisariopsis griseola Ferraris, que ocasiona la enfermedad mancha angular. Las selecciones en $\mathrm{F}_{5}$ se sembraron en Palmira, para determinar el rendimiento de grano bajo condiciones de sequía, de las selecciones superiores se tomó una planta individual para obtener una $\mathrm{F}_{6}$ uniforme (Steve Beebe, Com. Pers. Villar et al., 2013).

La línea de la cual se generó Frailescano, se introdujo a México en 2009, junto con otras 110 líneas y al estado de Chiapas en 2010, con el código SCN 6; a través, de un ensayo nacional de rendimiento de genotipos de frijol negro, para determinar su rendimiento, tolerancia a enfermedades y adaptación a suelo ácido. 


\section{Características agronómicas}

Las plantas de la variedad Frailescano son de hábito de crecimiento indeterminado, arbustivas y erectas, tipo II (Singh, 1982), con guías medianas, hojas pequeñas y una altura de planta promedio de $49.8 \mathrm{~cm}$. La floración se presenta en promedio a 38 días después de la siembra, la madurez fisiológica a 70 días y están listas para cosecharse a 80 días después de la siembra. Las flores son de color morado, tallos verdosos y vainas blancas en madurez fisiológica y de color blanco cremoso a la cosecha, con granos de color negro opaco, de tamaño pequeño y de forma ovoide.

Una de las características más sobresalientes de esta variedad es su resistencia a la enfermedad del mosaico amarillo dorado del frijol (BGYMV) y su adaptación a los suelos ácidos del trópico húmedo del estado de Chiapas (Villar et al., 2013). Por otra parte, al ser una variedad de ciclo precoz, su siembra en el ciclo de verano le permite al productor, cosechar el frijol en la época de canícula, para evitar problemas de exceso de humedad y en el ciclo de otoño-invierno, con humedad residual, escapar a la ocurrencia de periodos de sequía terminal, que comúnmente se presentan en la entidad (Figura 1) (López et al., 2002).

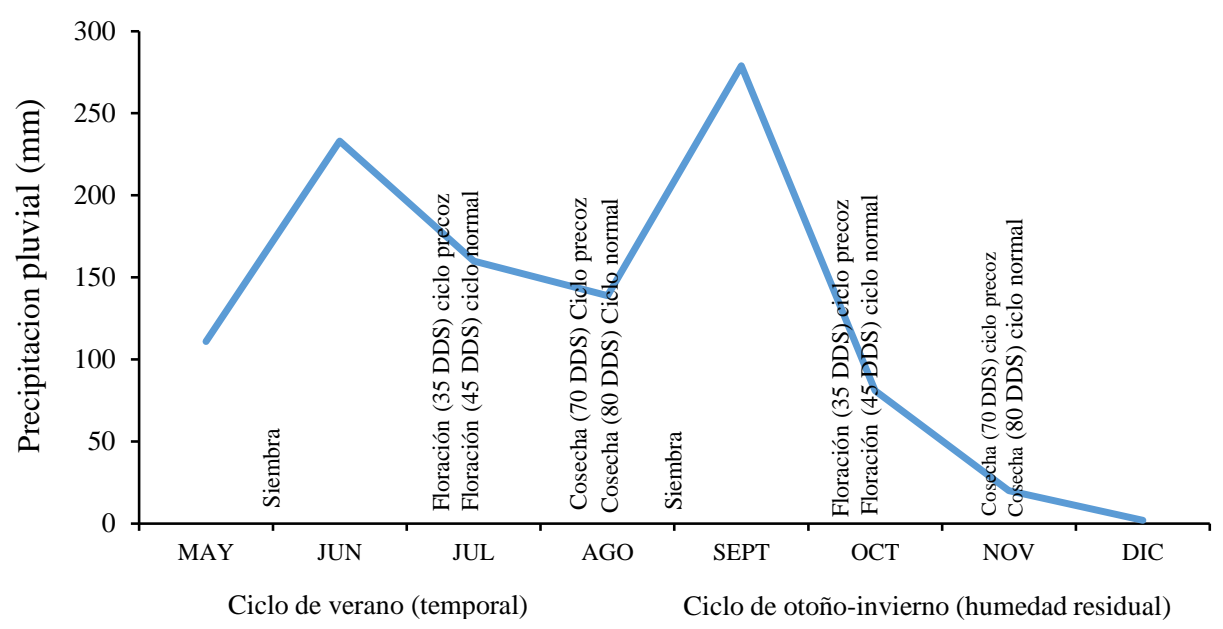

Figura 1. Esquema de la siembra de frijol con la variedad Frailescano (de ciclo precoz) y otra de uso común (de ciclo intermedio), en dos ciclos de cultivo.

\section{Comportamiento productivo}

Durante 2011 y 2012, la variedad Frailescano se comparó con las variedades comerciales Negro Papaloapan y Negro Comapa, utilizadas como testigos regionales, por su alto potencial de rendimiento y amplia adaptación en las áreas tropicales de Veracruz y Chiapas, a través de un ensayo uniforme de rendimiento regional que se condujo en ocho ambientes del estado de Chiapas, bajo condiciones de temporal, humedad residual y riego.

En el Cuadro 1 se muestra que, en la mayoría de los casos, la variedad Frailescano superó en rendimiento a los dos testigos comerciales. En condiciones de temporal, la variedad Frailescano superó en 17 y $28.4 \%$ el rendimiento de las variedades Negro Papaloapan y Negro Comapa, respectivamente. Con humedad residual, los porcentajes de superioridad de la variedad Frailescano, con respecto a estas mismas variedades fueron de 10.5 y $15.3 \%$ y cuando se utilizó riego de 15.8 y $13.2 \%$, respectivamente. De acuerdo con la prueba t de Student, el rendimiento 
promedio general de la variedad Frailescano (1 $\left.150 \mathrm{~kg} \mathrm{ha}^{-1}\right)$ fue estadísticamente similar al de la variedad Negro Papaloapan y significativamente superior al de la variedad Negro Comapa (Reyes, 1990).

Cuadro 1. Rendimiento de grano $\left(\mathrm{kg} \mathrm{ha}^{-1}\right)$ de la variedad Frailescano y dos variedades comerciales de frijol negro bajo tres condiciones de humedad en el centro de Chiapas.

\begin{tabular}{|c|c|c|c|c|}
\hline Condición de humedad/localidad & Ciclo/año & Frailescano & Negro Papaloapan & Negro Comapa \\
\hline \multicolumn{5}{|c|}{ Temporal } \\
\hline Ocozocoautla-1 & Verano 2012 & 1567 & 1326 & 1324 \\
\hline Ocozocoautla-2 & Verano 2012 & 508 & 312 & 335 \\
\hline Emiliano Zapata & Verano 2012 & 813 & 830 & 591 \\
\hline Promedio & & 963 & 823 & 750 \\
\hline Incremento respecto a testigos $(\%)$ & & & 17 & 28.4 \\
\hline \multicolumn{5}{|c|}{ Humedad residual } \\
\hline Ocozocoautla & O-I 2011 & 1760 & 1939 & 1776 \\
\hline Ocozocoautla-1 & O-I 2012 & 699 & 558 & 459 \\
\hline Ocozocoautla-2 & O-I 2012 & 765 & 317 & 514 \\
\hline La Unión & O-I 2012 & 1260 & 1243 & 1138 \\
\hline Promedio & & 1121 & 1014 & 972 \\
\hline Incremento respecto a testigos $(\%)$ & & & 10.5 & 15.3 \\
\hline \multicolumn{5}{|c|}{ Riego } \\
\hline Ocozocoautla & I-P 2012 & 1828 & 1578 & 1614 \\
\hline Incremento respecto a testigos $(\%)$ & & & 15.8 & 13.2 \\
\hline Promedio general & & $1150^{* *}$ & $1012.9 \mathrm{~ns}$ & 968.9 \\
\hline Incremento respecto a testigos $(\%)$ & & & 13.5 & 18.7 \\
\hline
\end{tabular}

$\mathrm{V}=$ ciclo de verano; OI= ciclo de otoño-invierno; $\mathrm{IP}=$ ciclo de invierno-primavera; ${ }^{* *}=$ diferencia significativa $(\mathrm{t}-$ Student, $p<0.01) ; \mathrm{ns}=$ diferencias de promedios no significativas.

Estos resultados experimentales indican que, en el centro del estado de Chiapas, la variedad Frailescano tiene similar potencial de rendimiento y adaptación que la variedad Negro Papaloapan y superior a la de Negro Comapa; ambas variedades fueron desarrolladas por el Programa de Frijol del INIFAP para el sureste de México (López et al., 2007; 2012).

\section{Reacción al BGYMV}

En el ciclo de verano de 2012, bajo condiciones de temporal, en la localidad de Emiliano Zapata, municipio de Villa Corzo, Chiapas, se evaluó la reacción de la variedad Frailescano, junto con las variedades Negro Papaloapan y Negro Comapa, a la incidencia de mosaico amarillo dorado del frijol (BGYMV), a los 20, 28 y 35 días después de la siembra. Las variedades Negro Papaloapan y Negro Comapa mostraron síntomas de la enfermedad desde los 20 días después de la siembra, cuyo 
porcentaje de incidencia en la última fecha de evaluación fue de 40 y $23 \%$, respectivamente, en tanto que, la variedad Frailescano sólo presentó síntomas débiles de mosaico amarillo dorado, con un porcentaje de incidencia de 13\%, hasta los 35 días después de la siembra (Schoonhoven-Van y Pastor-Corrales, 1987) (Cuadro 2).

Por lo anterior, en áreas de cultivo con alta incidencia de mosquita blanca (Bemisia tabaci), vector del BGYMV, la variedad Frailescano es mejor alternativa de siembra que las variedades Negro Papaloapan y Negro Comapa.

Cuadro 2. Porcentaje de incidencia del mosaico amarillo dorado en la variedad Frailescano y dos variedades comerciales de frijol, en tres fechas de evaluación de la enfermedad.

\begin{tabular}{cccc}
\hline \multirow{2}{*}{ Variedad } & \multicolumn{3}{c}{ Fechas de evaluación (dds) } \\
\cline { 2 - 4 } & 20 & 28 & 35 \\
\hline Frailescano & 0 & 0 & 13 \\
Negro Papaloapan & 12 & 29 & 40 \\
Negro Comapa & 12 & 13 & 23 \\
\hline
\end{tabular}

dds= días después de la siembra.

\section{Adaptación a suelo ácido}

En 2012, en la localidad Emiliano Zapata, se comparó la respuesta productiva de la variedad Frailescano con el Criollo Veracruzano, de uso común en el centro de Chiapas (Villar et al., 2013), en un suelo ácido (con pH de 4 y saturación de aluminio de $20 \%$ ), sin y con aplicación de $1 \mathrm{t} \mathrm{ha}^{-1}$ de cal agrícola. Tanto en condiciones naturales de acidez edáfica, como con aplicación de cal, la variedad Frailescano superó en más de $96 \%$ el rendimiento del Criollo Veracruzano. La reducción de rendimiento, debido al estrés por suelo ácido, fue menor en la variedad Frailescano, que en el Criollo Veracruzano (Cuadro 3). Estos resultados indican que, en los suelos ácidos del centro de Chiapas, la variedad Frailescano, es mejor alternativa de siembra que el Criollo Veracruzano.

Cuadro 3. Rendimiento de grano $\left(\mathrm{kg} \mathrm{ha}^{-1}\right)$ de la variedad Frailescano y el criollo Veracruzano, en un suelo ácido, sin y con aplicación de cal agrícola, en el centro de Chiapas.

\begin{tabular}{cccc}
\hline \multirow{2}{*}{ Variedad } & \multicolumn{2}{c}{ Condición de suelo } & \multirow{2}{*}{ Reducción (\%) } \\
\cline { 2 - 3 } & Con $1 \mathrm{t} \mathrm{ha}^{-1}$ de cal & Sin cal & \\
\hline Frailescano & 1000.5 & 861.7 & 13.87 \\
Criollo Veracruzano & 509 & 428 & 15.91 \\
Incremento con respecto al testigo (\%) & 96.6 & 101.3 & \\
\hline
\end{tabular}

\section{Validación de la variedad Frailescano}

De 2014 a 2016, la variedad Frailescano se validó en terrenos de agricultores, en cinco ambientes del estado de Chiapas (dos bajo condiciones de riego y tres con la humedad residual almacenada en el suelo y las lluvias que ocurrieron durante el ciclo del cultivo). En todos los casos, se utilizó como testigo al criollo Vaina Negra de uso común en la entidad. En las dos condiciones de humedad, la variedad Frailescano superó en rendimiento al criollo regional. Cabe resaltar que, en 
la localidad de Alfonso Moguel, en otoño-invierno de 2016, hubo sequía severa durante la fase reproductiva del cultivo, bajo estas condiciones restrictivas de humedad, la variedad Frailescano obtuvo 58\% más de rendimiento de grano que el criollo Vaina Negra.

EI rendimiento promedio general de la variedad Frailescano fue de $1438 \mathrm{~kg} \mathrm{ha}{ }^{-1}$, significativamente superior (51.1\%) al obtenido por el criollo regional (Cuadro 4). Estos resultados permitieron confirmar a nivel semi-comercial y bajo las condiciones de suelo, humedad y manejo de los agricultores, la mayor capacidad productiva de la variedad Frailescano, en comparación con los materiales criollos comúnmente utilizados en el estado de Chiapas.

Cuadro 4. Rendimiento de grano $\left(\mathrm{t} \mathrm{ha}^{-1}\right)$ de dos variedades de frijol en parcelas de validación en cinco ambientes de Chiapas.

\begin{tabular}{lccccc} 
Localidad/municipio & \multicolumn{2}{c}{ Ciclo y año } & $\begin{array}{c}\text { Ambiente de } \\
\text { producción }\end{array}$ & \multicolumn{3}{c}{ Frailescano Vaina Negra Incrmento } \\
& \multicolumn{4}{c}{ (\%) } \\
\hline Jiquipilas, Jiquipilas & OI-2014 & Riego & 2900 & 1800 & 61.1 \\
Independencia, Jiquipilas & IP-2014 & Riego & 1600 & 950 & 68.4 \\
Espinal de Morelos, Ocozocoautla & OI-2014 & HR & 850 & 750 & 13.3 \\
Alfonso Moguel, Ocozocoautla & OI-2014 & HR & 1350 & 950 & 42.1 \\
Alfonso Moguel, Ocozocoautla & OI-2016 & HR & 490 & 310 & 58.1 \\
Promedio & & & $1438^{*}$ & 952 & 51.1 \\
\hline
\end{tabular}

$\mathrm{OI}=$ ciclo de otoño-invierno. $\mathrm{IP}=$ ciclo de invierno-primavera; $\mathrm{HR}=$ condición de humedad residual; ${ }^{1}=$ porcentaje de incremento de rendimiento respecto al testigo; ${ }^{*}=$ diferencia significativa (t- Student, $p<0.05$ ).

\section{Condiciones agroecológicas}

La variedad Frailescano tiene adaptación en las áreas tropicales del estado de Chiapas, así como en regiones con condiciones ambientales similares del sureste de México. Puede sembrarse en el ciclo de verano, bajo condiciones de temporal y de otoño-invierno con humedad residual, en áreas con altitudes que van desde 0 hasta $1200 \mathrm{~m}$, precipitación pluvial de al menos $350 \mathrm{~mm}$ durante el ciclo del cultivo y temperatura media anual de entre 24 y $26^{\circ} \mathrm{C}$.

También puede sembrarse en áreas que disponen de agua y equipo para riego, donde se asegure la disponibilidad de una lámina de agua de alrededor de $350 \mathrm{~mm}$, distribuidos en seis o siete riegos, con un intervalo de entre 10 y 15 días cada uno durante el ciclo del cultivo, dependiendo de las condiciones de humedad en el suelo y la temperatura del ambiente (López et al., 2002; Ruíz et al., 2013).

\section{Disponibilidad de semilla}

En el Campo Experimental Centro de Chiapas se dispone de semilla original de la variedad Frailescano, para producir semilla básica y registrada, en caso de que asociaciones de productores o empresas semilleras deseen adquirirla, para producir la semilla certificada. El número de registro de esta variedad en el Catálogo Nacional de Variedades Vegetales del SNICS, para la producción de semilla y uso comercial es: FRI-088-060314. 


\section{Conclusiones}

La variedad Frailescano mostró un rendimiento promedio experimental de $1150 \mathrm{~kg} \mathrm{ha}^{-1}$, significativamente superior al de la variedad Negro Comapa, mayor tolerancia al mosaico amarillo dorado, que las variedades Negro Papaloapan y Negro Comapa y mejor adaptación en condiciones de suelo ácido y sequía severa, que los criollos regionales.

Esta variedad puede cultivarse en áreas tropicales y subtropicales del estado de Chiapas y regiones con condiciones similares del sureste de México, con una altitud hasta de $1200 \mathrm{~m}$, bajo condiciones de temporal, humedad residual y riego. Su precocidad le permite al productor disminuir riesgos de pérdida de rendimiento por la ocurrencia de periodos de sequía terminal y su grano negro opaco, de tamaño pequeño, reúne las características de tipo de frijol que demandan los productores y consumidores en el estado de Chiapas.

\section{Literatura citada}

López, S. E.; Acosta, G. J. A.; Cumplan, G. J.; Cano, R. O.; Villar, S. B. y Becerra, L. E. N. 2002. Adaptación de genotipos de frijol común en la región tropical húmeda de México. Agric. Téc. Méx. 28(1):35-42.

López, E.; Tosquy, O. H.; Villar, B.; Becerra, E. N. y Ugalde, F. J. 2003. Adaptación, resistencia múltiple a enfermedades y tolerancia a suelos ácidos en genotipos de frijol. Agron. Mesoam. 14(2):151-155.

López, S. E.; Tosquy, V. O. H.; Villar, S. B.; Becerra, L. E. N.; Ugalde, A. F. J. y Cumpián, G. J. 2006. Adaptabilidad de genotipos de frijol resistentes a enfermedades y a suelos ácidos. Rev. Fitotec. Mex. 29(1):33-39.

López, S. E.; Tosquy, V. O. H.; Villar, S. B.; Cumpián, G. J.; Ugalde, A. F. J. y Becerra, L. E. N. 2007. Negro Papaloapan, nuevo cultivar de frijol para las áreas tropicales de México. Agric. Téc. Méx. 33(3):257-267.

López, S. E.; Tosquy, V. O. H.; Jiménez, H. Y.; Salinas, P. R. A.; Villar, S. B. y Acosta, G. J. A. 2012. Rendimiento y adaptación de la variedad de frijol 'Negro Comapa' en dos regiones de México. Rev. Fitotec. Mex. 35(4):309-315.

Reyes, C. P. 1990. Diseño de experimentos aplicados agronomía, biología, química, industrias, ciencias sociales y ciencias de la salud. $3^{\mathrm{a}}$ (Ed.). Trillas. México, DF. 348 p.

Rosas, J. C.; Hernández, J. C. and Araya, R. 2003. Registration of Bribri'small red bean (race Mesoamerica). Crop Sci. 43(1):430-431.

Ruíz, C. J. A.; Medina, G. G.; González, A. I. J.; Flores, L. H. E.; Ramírez, O. G.; Ortiz, T. C.; Byerly, M. K. F. y Martínez, P. R. A. 2013. Requerimientos agroecológicos de cultivos. INIFAP. CIRPAC. Campo Experimental Centro Altos de Jalisco. Tepatitlán de Morelos, Jalisco, México. Libro técnico núm. 3. 564 p.

Schoonhoven-Van, A. y Pastor-Corrales, M. A. 1987. Sistema estándar para la evaluación de germoplasma de frijol. van Schoonhoven, A. y Pastor-Corrales, M. A. (Comps.). Centro Internacional de Agricultura Tropical (CIAT). Cali, Colombia. 56 p.

SIAP. 2020. Servicio de Información Agroalimentaria y Pesquera. Avance de siembras y cosechas. Resumen por estado. SIAP. Gobierno de México. http://infosiap.siap.gob.mx:8080/ agricola_siap_gobmx/ResumenProducto.do. 
Singh, S. P. 1982. A key for identification of different growth habits of Phaseolus vulgaris L. The XXV Ann. Rep. Bean Improv. Coop. 92-95 pp.

Tosquy, V. O. H.; López, S. E.; Esqueda, E. V. A.; Acosta, G. J. A.; Ugalde, A. F. J. y Villar, S. B. 2012. Rendimiento y reacción a enfermedades de genotipos de frijol en condiciones de temporal y humedad residual. Rev. Mex. Cienc. Agríc. 3(4):727-737.

Villar, S. B. y López, S. E. 1993. Negro INIFAP: nueva variedad de frijol para Chiapas y regiones tropicales similares. Rev. Fitotec. Mex. 16(2):208-209.

Villar, S. B. y López, S. E. 2003. Negro Grijalva. Nueva variedad de frijol para Chiapas y regiones similares. SAGARPA. INIFAP. Campo Experimental Centro de Chiapas. Ocozocoautla, Chis., México. Folleto técnico núm. 2. 22 p.

Villar, S. B.; López, S. E.; Tosquy, V. O. H. y Ugalde, A. F. J. 2010. Rojo INIFAP, nueva variedad de frijol de grano rojo para el trópico de México. Rev. Mex. Cienc. Agríc. 1(5):681-686.

Villar, S. B.; López, S. E.; Tosquy, V. O. H.; Cruz, Ch. F. J. y Acosta, G. J. A. 2013. Frailescano. Nueva variedad de frijol para el estado de Chiapas y regiones bajo condiciones similares. SAGARPA. INIFAP. CIRPAS. Campo Experimental Centro de Chiapas. Ocozocoautla, Chis., México. Folleto técnico núm. 18. 25 p.

Villar, S. B.; Tosquy, V. O. H. y López, S. E. 2017. Rendimiento y adaptación de la variedad de frijol Rojo INIFAP (Phaseolus vulgaris L.) en Chiapas, México. Agroproductividad. 10(9):64-70.

Zetina, L. R.; Pastrana, A. L.; Romero, M. J. y Jiménez, Ch. J. A. 2002. Manejo de suelos ácidos para la región tropical húmeda de México. $1^{\text {ra. }}$ (Ed.). INIFAP. CIRGOC. Campos Experimentales del Papaloapan y Huimanguillo. México, DF. Libro técnico núm. 10. $170 \mathrm{p}$. 